\title{
Internet use among urban Malaysians: Network diversity effects
}

\author{
Antoon De Rycker ${ }^{1, *}$, Nicole Yang Lai Fong ${ }^{1}$, Catherine Lee Cheng Ean ${ }^{2}$, and \\ Ramachandran Ponnan ${ }^{1}$ \\ ${ }^{1}$ Taylor's University, School of Communication, 47500 Subang Jaya, Malaysia \\ ${ }^{2}$ Sunway University, Department of Communication and Liberal Arts, 46150 Subang Jaya, Malaysia
}

\begin{abstract}
This study examines social network diversity in urban areas relative to residents' usage of information and communication technologies (ICTs). Individual-level variation in social network diversity was measured using position generator data collected as part of a survey conducted in Malaysia's Klang Valley $(\mathrm{N}=808)$. Regression analyses were performed to assess the extent to which network diversity is related to ICTs. We find that most ICTs have a negative effect on diversity. Only frequent use of the Internet at work, mobile access to the Internet, and reading online news or blogs contribute positively to diversity. Findings support both a tendency toward 'networked individualism' and the more recent 'glocalization' thesis that some ICTs may also afford participation within local space rather than only across distant space.
\end{abstract}

\section{Introduction}

The growth of social media platforms (e.g., Facebook) has renewed interest in the analysis of sociality through social networks, focussing on network diversification and social capital effects (e.g., [1-2]). The central question concerns the ways in which digital technologies influence social relations and Internet users' personal networks.

A personal or 'egocentric' network is generally defined as 'the network surrounding one node, known as the ego' [3]. For different egos, this set of socially relevant 'alters' may vary in diversity, or within-group homogeneity [4-5]. As a social construct, it corresponds to the notion of variety: the extent to which individuals differ from one another on certain sociodemographic, behavioral or intrapersonal attributes (e.g., occupation or education but also whether someone plays volleyball, likes gardening or has visited Paris) [6-7]. Given the wide range of attributes in which individuals can differ, various operationalizations of the same 'social network diversity' construct is possible.

Our main purpose is to identify the social network affordances of new media among urban Malaysians. Secondly, we would like to find out whether the Malaysian findings support any of the main arguments put forward in connection with the role of ICTs, new media, and digital culture in contemporary society. For example, relationships between the Internet and social network diversity have been found to be either direct or mediated, either

* Corresponding author: antoon.derycker@taylors.edu.my 
positive or negative; considerable attention has been paid to so-called 'networked individuals' and their lack of participation in traditional local settings (e.g., public libraries and coffee shops) [8]. It is important to examine these and similar statements for different populations and to see whether findings can put some of the existing Western scholarship in a broader perspective. Given the relative paucity of social network research on Malaysia (e.g., [9-11]), our study also seeks to contribute to a better understanding of the social texture of urban Malaysian society.

\section{The Internet and social network diversity}

Researchers examining Internet use and social networks are in agreement that as a conceptual category, 'Internet use' is too undifferentiated to capture the unique 'social affordances' [12-14] of each digital media or device. Facebook, for example, can be used to meet new people, to find people to date, to keep in touch with old friends, to check out people a person has met socially, etc.; all of these utilities, however, have different social network implications [1]. By looking at the detail of social media, researchers are now able to make more precise, more qualified, and less sweeping statements than the either/or positions that characterized some of the earlier research and social commentary [15].

The detailed findings can be related to the more general arguments that have been developed in the literature. A survey of two decades of Internet research identifies three main theories: (1) the Internet helps form close and meaningful relationships around shared common interests, both globally and locally; (2) the Internet isolates and alienates people from their communities, negatively impacting social network diversity and social capital; (3) the Internet supplements other forms of interaction and communication, serving 'an additive role' [1]. The third perspective corresponds to a view of the Internet as enhancing community instead of weakening or transforming it [16]. However, different explanations of the use and impact of the Internet need not be mutually exclusive: 'they all may be happening simultaneously', and also, different ICTs 'may have contradictory relationships' [4]. Facebook and other social networking services, for example, contribute to social network diversity directly as well as indirectly through neighborhood ties; yet, while the former effect is positive (more diversity), the latter is negative (less diversity). These opposite findings are consistent, however, with earlier studies that relate social networking sites to higher aggregate levels of network diversity but reduced neighborhood involvement $[17,4]$.

The second theoretical perspective - isolation and alienation - can be related to early Internet scholarship such as the 'networked individualism' argument [18]. Under this view, the Internet provides a new social setting for the formation and maintenance of social ties. However, when more time is spent interacting online globally than in offline groups or social settings, this creates a transformational shift from so-called place-based to personbased sociality. Personal networks become dichotomous, i.e., 'online and global' versus 'offline and local.' Although 'networked individuals' may form many new ties online [19], those ties are not necessarily diverse. A plausible explanation is that the Internet may 'function more like the private home than a public space - building large homophilous networks at the expense of diverse ties' [4]. Thus, the replacement of 'densely-knit and tightly-bounded groups' by 'sparsely-knit and loosely-bounded networks' [20] would not only increase individualism and privatism but also decrease social network diversity overall.

Networked individualism is not the only theory that has been proposed when attempting to describe online behaviour. Within the long-standing debate about the effect of the Internet on personal networks, Hampton and his co-researchers have recently argued in favour of what they refer to as 'glocalization.' [4]. Originally, the term referred to 'the 
intermingling of intensive local and extensive long-distance relationships;' however, glocalization is now used within media and communication research to refer to 'the combination of global connectivity and local activity' [15]. Whenever the Internet is adopted by a critical mass of individuals repeatedly interacting within a shared local setting (e.g., the neighborhood or workplace), it will increase awareness that the Internet affords communication within local space, i.e., with social actors in the vicinity rather than across distant space [21-22]. In other words, glocalization describes the phenomenon that individuals can maintain multiple, partially interlocking and differentially diverse personal networks that are 'offline and local,' 'online and global' but also 'online and local.'

Note that the present study is not aimed at developing a new way of thinking about the relationship between Internet use and the diversity of social networks. Also, networked individualism, glocalization, and other 'substantive theories' are restricted in descriptive accuracy and explanatory scope to a particular social setting [23]. Still, and as observed in Section 1, our findings can be used to test or refine existing theories, with urban Malaysia as a comparative case [24].

\section{Methodology}

\subsection{Data}

The dataset for this analysis is based on the survey responses of 808 adults, aged 31 to 55, living in the Klang Valley. The Klang Valley is Malaysia's most densely populated, most heavily urbanized, and most ethnically diverse region [25]. The area includes the country's capital city, Kuala Lumpur, with a population of around 1.6 million; the total population of the Klang Valley is estimated at 7.5 million [25]. For more details about the sampling breakdown, see [10].

The questionnaire is based on a previously validated and published survey instrument $[4,26]$. Minor adjustments were made to make the items and responses suitable for use in Malaysia (and thus maintain content validity). First, two occupations, i.e., 'Congressman' and 'middle-school teacher,' were replaced by respectively 'Member of Parliament' and 'secondary school teacher.' Second, when considering the response options for employment status, we separated 'other' into 'self-employed: professional' (e.g., architect) and 'selfemployed: business owner' (e.g., shopkeeper). This distinction was introduced to reflect the Klang Valley's small-scale, entrepreneurial economy more accurately.

Note that neither of these changes consists in rewordings, reframing of the original questions or adopting a new scoring system. Phrases like 'Member of Parliament' do not introduce comprehension issues either; on the contrary, they are simple, straightforward, and appropriate for the reading level of the respondents [27]. Still, to minimize the risk that our localized version would affect the instrument's accuracy and validity, a pilot test was conducted among colleagues prior to administration.

\subsection{Measures}

The dependent (outcome) variable of interest is social network diversity. The independent (predictor) variable used to account for variability in the level of social network diversity is respondents' self-reported use of various ICTs. Selection and operationalization of the key variables were modeled after [4]. 


\subsubsection{Social network diversity}

As in many social network studies, a position generator instrument was used to measure the dependent variable (e.g. [28]). Respondents were asked to indicate whether or not they know someone in a specified occupation, and if so, to assess the closeness of the relationship $(1=$ no one, $2=$ someone but not close at all, $3=$ less close but still important, $4=$ close, $5=$ very close). The more ties someone has to other social positions, and the stronger those ties are, the more diverse (or heterogeneous) a person's egocentric network. The generator includes occupations that vary in socioeconomic standing; for the complete list of 22, see [4]. but also race and religion. In regard to race, the main reason is that Malaysia can be characterized as 'a society of multi-ethnicities' in which different cultures co-exist without necessarily blending into each other [29].

As in [4], responses to the generator were subsequently combined into a composite scale measure; note that this increases the reliability and validity of the instrument [24]. Specifically, an additive index was calculated based on 29 items: the 22 occupations (ranging in prestige from security guard to Chief Executive Officer), the three main races (Malay, Chinese, and Indian) and the four main religions (Muslim, Buddhist, Hindu, and Christian). The mean social network diversity score was $2.57(\mathrm{SD}=.622)$.

\subsubsection{Information and communication technologies}

To measure Internet use, survey respondents were first asked how often they went online (1 = every day, $2=$ several times a week, $3=$ once or twice a week, $4=$ once or twice a month, $5=$ less than once a month). Next, they were presented with a series of dichotomous questions about whether or not they had Internet access at home and at work, and if so, what types of access were available (e.g., digital subscriber line). Finally, given this study's focus on the network diversity affordances of different ICTs, we asked respondents whether or not they engaged in a variety of online activities (a total of twelve) such as instant messaging, sharing digital photos, social networking, and banking.

The mean frequency of Internet use was 1.50 (i.e., going online several times a week up to at least once every day) with a standard deviation of 1.064. A majority of respondents uses the Internet from home $(\mathrm{n}=532,65.8 \%)$, compared with a slightly lower number for Internet use at work $(\mathrm{n}=473,58.5 \%)$; for both, wireless connection is the most common access method. Most respondents engage in five to six online activities $(n=227,28.1 \%)$, with very few reporting no activities $(\mathrm{n}=36,4.5 \%)$ or all twelve $(\mathrm{n}=38,4.7 \%)$ (data not shown).

\subsection{Analysis procedure}

The data were analysed by running a series of ordinary least squares (OLS) regression analyses. Generally, when studying the multivariate nature of the social world, multiple regression analysis is the tool of choice but only when all variables are continuous; in the current study, however, only the outcome variable is continuous while each of the ICTrelated predictor variables is either categorical or rank. For more justification, see also [24]. Following [4], we used unstandardized regression coefficients to capture the associations instead of the more usual standardized ones.

\section{Findings and discussion}

A first major finding is that different types of ICT contribute differently to social network diversity. The more specific results of the regressions have been summarized in Table 1 . 
Table 1. Ordinary Least Squares (OLS) regressions of social network diversity outcomes $(\mathrm{N}=808)$.

\begin{tabular}{|l|c|}
\hline Regression coefficients & Network diversity \\
\hline Constant & $2.639^{* * *}$ \\
\hline Frequency of Internet use & .045 \\
\hline Frequent Internet use at home & $-.107^{*}$ \\
\hline Internet access at home & $-.081^{* *}$ \\
\hline Frequent Internet use at work & .003 \\
\hline Internet access at work & .019 \\
\hline Internet access through mobile devices & -.085 \\
\hline Instant messaging & .133 \\
\hline Sending emails & $-.244^{* * *}$ \\
\hline Blogging & -.015 \\
\hline Posting messages (e.g., forum) & $-.168^{* *}$ \\
\hline Sharing digital photos & -.002 \\
\hline Exploring an interest or hobby & .091 \\
\hline Purchasing (e.g., music and books) & $-.134^{*}$ \\
\hline Playing video games and MPGs & $-.173^{* *}$ \\
\hline Social networking (e.g., Facebook) & .038 \\
\hline Reading news or blog posts & .035 \\
\hline Banking & -.023 \\
\hline Stock transactions & .077 \\
\hline & \\
\hline
\end{tabular}

For the majority of ICTs and online activities, we find that the relationship to social network diversity is negative or that it has no statistical significance. Among the statistically significant relationships of diversity, one third are negative: social network diversity is lower among respondents who have Internet at home, access the Internet from home every day, send emails, post messages, buy over the Internet, and play online (multiplayer) video games. People who send emails, for example, have networks that are, on average, $.244 \mathrm{SD}$ less diverse than those who do not (or do so less frequently). As for the remaining online activities, no statistically significant linear dependence of diversity on these various regressors was detected. They include sending instant messages, blogging, sharing digital photos (e.g., Flickr), exploring an interest or hobby, banking, stock transactions, and perhaps unexpectedly - considering [4] - social networking services (e.g., Facebook or Weibo). Although ranked second after instant messaging $(\mathrm{n}=635,78.6 \%)$, it could not be established whether this type of social media use contributes to explaining the variability in personal network diversity. 
Taken together, our ICT results suggest that urban Malaysians' online behaviour has created a new social setting that is in (partial) competition with traditional ones but that has a higher likelihood of reducing social network diversity. Within the theory of 'networked individualism' [18], this would then suggest that urban Malaysians function socially within dichotomous personal networks rather than 'glocalized' ones. Social network diversity among urban Malaysians is medium to low $(\mathrm{M}=2.57$, on a scale from one to five). When discounting racial/ethnic and religious diversity responses and after conversion into a binary categorical variable $(0=$ no one, $1=$ at least one person), we arrive at a social network diversity index of $\mathrm{M}=11.71$ ( $\mathrm{SD}=5.59)$; this is only slightly higher than $\mathrm{M}=$ $9.25(\mathrm{SD}=5.30)$ found in the US ([4]). This figure would be difficult to account for within a 'glocal' perspective: for one thing, percentages for Internet access and frequency of use in the Klang Valley are high (see Table 1), and for another, Malaysia's overall ratings for Internet affordability, skills, and individual usage are above average compared with other upper-middle-income countries ([30]). These results would thus support Putnam's original 'time displacement' hypothesis ([31]), at least, as applied to the Internet and today's digital technologies ([32]).

Despite these tendencies, the data do not point to a deeper, more structural shift toward 'networked individualism.' Within the set of negative relationships, coefficients are low, ranging from .081 SD (frequent Internet use at home) to .244 SD (sending emails). What is striking is that in urban Malaysia, affordances for higher network diversity are less in evidence than in the US (e.g., [4]). For example, in the US, the use of social networking services contributes directly to egocentric network diversity but in urban Malaysia, respondents who use social media (78.6\% of the sample), the regression analysis does not allow us to draw any conclusions.

Explanations for the mixed results and the similarities with, and differences from, the US findings may be looked for in (1) the specificity of the individual ICTs examined in this study, (2) respondents' 'network practices' [33], as in, for example, the amount of active communication on Facebook [15], (3) properties of their egocentric social networks other than diversity such as size, density or centrality, and (4) structural aspects of contemporary Malaysian society. Given the large number of variables and combinations among them, we will not explore this further in the present study. The main purpose has been to gain insight into the 'bigger picture' relating digital media (and devices) and egocentric network diversity. Further examination of the many individual survey findings will allow a deeper, more nuanced understanding of social network diversity among urban Malaysians but this will be reported in a separate publication.

This study was supported by Grant FRGS/1/2012/SS09/TAYLOR/03/1 from the Malaysian Ministry of Higher Education, 2013-2015.

\section{References}

1. N. B. Ellison, C. Steinfeld, C. Lampe. Journal of Computer-Mediated Communication 12, 3: 1143-1168 (2007)

2. A. L. Gonzales. Communication Research 42: 1-20 (2015)

3. A. Marin, B. Wellman. The Sage handbook of social network analysis. Thousand Oaks, CA: Sage Publications, 11-25 (2011)

4. K. N. Hampton, C. J. Lee, E. J. New Media and Society 13, 7: 1-19 (2011)

5. J. Porter, M. O. Emerson. Social Science Quarterly 94, 3: 732-757 (2013)

6. D. A. Harrison K. J. Klein. Academy of Management Review 32, 4: 1199-1228 (2007)

7. M. McPherson, L. Smith-Lovin, J. M. Cook. Annual Review of Sociology 27, 1: 415$444(2001)$ 
8. L. Rainie, B. Wellman. Cambridge, MA: MIT Press. (2012)

9. H. M. Adnan, S. R. Mavi. Asian Social Science 11, 15: 1-9 (2015)

10. A. De Rycker, L. F. Yang, C. E. Lee, R. Ponnan, V. S. Lokasundari. International Journal of Asia Pacific Studies 11:61-92 (2015)

11. W. M. W. Jaafar. The Macrotheme Review 3, 2: 170-187 (2014)

12. E. Bradner. Paper presented at the CHI 2001 Conference on Human Factors in Computing Systems, Seattle, WA. (2001)

13. B. Wellman. In: K Christensen, D Levinson (Eds.), Encyclopedia of community: From the village to the virtual world. Thousand Oaks, CA: Sage, 560-563. (2003)

14. S. Zhao. Journal of Computer-Mediated Communication 11, 3: 844-862 (2006)

15. A. Bohn, C. Buchta, K. Hornik, P. Mair. Social Networks 37: 29-41 (2014)

16. B. Wellman, J. Boase, W. H. Chen. IT \& Society 1:151-65 (2002)

17. K. N. Hampton, L. F. Sessions, E. J. Her, L. Rainie. Pew Research Center. Washington, DC. (2009)

18. B. Wellman. International Journal for Urban and Regional Research 25, 2: 227-252 (2001)

19. C. Haythornthwaite. Information, Communication \& Society 8, 2: 125-147 (2005).

20. B. Wellman, A. Quan-Haas, J. Boase, W. Chen, K. Hampton, I. Díaz, K. Miyata. Journal of Computer-Mediated Communication 8.

21. K. N. Hampton. American Behavioral Scientist 53, 8: 1111-1132 (2010)

22. K. N. Hampton KN, B. Wellman. City and Community 2, 3: 277-311 (2003)

23. J. W. Creswell. Research design: Qualitative, quantitative and mixed methods approaches (4th ed). London: Sage (2014)

24. W. P. Vogt, E. R. Vogt, D. C. Gardner, L. M. Haeffele. Selecting the right analyses for your data: Quantitative, qualitative, and mixed methods. New York: The Guilford Press (2014)

25. National Census. Population distribution and basic demographic characteristic report 2010 (updated 5 August 2011). Department of Statistics Malaysia. Available at: http://www.statistics.gov.my (2010)

26. N. Lin, B. H. Erickson BH (Eds.). Social capital: An international research program. New York: Oxford University Press (2008)

27. L. A. Clark, D. Watson. Constructing validity: Basic issues in objective scale development. Psychological Assessment 7, 3: 309-319 (1995)

28. N. Lin, Y. C. Fu, R. M. Hsung. The position generator: Measurement techniques for investigations of social capital. In: N. Lin, K. Cook, R. Burt (Eds.). Social capital: Theory and research. New York: Aldine de Gruyter, 57-81. (2001)

29. A. R. Embong. State-led modernization and the new middle class in Malaysia. Basingstoke: Palgrave. (2002)

30. S. Dutta, T. Geiger, B. Lanvin (Eds.). The global information technology report 2015: ICTS for inclusive growth. Geneva: World Economic Forum and INSEAD. (2015)

31. R. D. Putnam. Tuning in, tuning out: The strange disappearance of social capital in America. Political Science and Politics 28, 4: 664-683 (1995)

32. M. Kestnbaum, J. P. Robinson, A. Neustadtl, A. S. Alvarez. IT and Society 1, 1: 21-37 (2002)

33. B. Hollstein. Qualitative approaches. In: J. Scott, P. J. Carrington (Eds.), The Sage handbook of social network analysis. Thousand Oaks, CA: Sage Publications, 405416. (2011) 